\title{
Longitudinal-to-transverse mapping for femtosecond electron bunch length measurement
}

\author{
Dao Xiang and Yuantao Ding \\ SLAC National Accelerator Laboratory, Menlo Park, California 94025, USA
}

(Received 26 April 2010; published 1 September 2010)

\begin{abstract}
A longitudinal-to-transverse mapping technique is proposed to measure the length and temporal profile of ultrashort electron bunches. In this scheme a special chicane and a radio-frequency deflecting cavity are used to transform the beam's longitudinal distribution into angular distribution which is further converted to transverse distribution after a parallel-to-point imaging beam line. With this technique, the temporal profile of the electron beam is exactly mapped to the transverse profile. This makes it possible to measure ultrashort electron bunch length with a resolution well beyond 1 femtosecond.
\end{abstract}

DOI: 10.1103/PhysRevSTAB.13.094001

PACS numbers: 29.27.Fh, 41.60.Cr

\section{INTRODUCTION}

Ultrashort electron bunches with an rms length of $\sim 1$ femtosecond (fs) or less have wide applications. They can be used to generate ultrashort $\mathrm{x}$-ray pulses in free electron lasers (FELs) [1-3] that may enable the time-resolved study of electronic dynamics, which can open up many new regimes in ultrafast sciences. It is also envisioned that ultrashort electron bunches may excite $\sim \mathrm{TeV} / \mathrm{m}$ wakefields for plasma wakefield acceleration [4,5] and high field physics studies. Recent success of using $20 \mathrm{pC}$ electron beam to drive an X-ray FEL at the Linac Coherent Light Source (LCLS) [6,7] has stimulated worldwide interest in using low charge beam $(1 \sim 20 \mathrm{pC})$ to generate ultrashort $\mathrm{x}$-ray pulses (0.1 fs-10 fs) in FELs $[8,9]$.

Accurate measurement of the length and temporal profile of the ultrashort electron bunch is essential for understanding the physics associated with the bunch compression and transportation. However, the shorter and shorter electron bunch greatly challenges the present beam diagnostic methods. Several techniques have been developed in the past decade to measure the length and temporal profile of short bunches (for a recent review, see Ref. [10]). So far the highest resolution in bunch length measurement is about 10-20 fs with a radio-frequency (rf) deflecting cavity [11,12]. Further improvement of the resolution of a deflecting cavity can be achieved by increasing the deflection voltage and reducing the wavelength of the deflection field. An optical deflector using a high power laser with a transverse mode may extend the resolution to $\sim 1$ fs. However, all these methods have the fundamental drawback that the resolution is limited by the beam's intrinsic transverse emittance.

In this paper, we propose a novel scheme to break the fs time barrier in bunch length measurement by overcoming the fundamental resolution limit arising from the transverse emittance. In this scheme a special chicane together with a standard rf deflecting cavity first maps the beam's temporal distribution exactly to the beam's angular distribution. Then a parallel-to-point imaging beam line converts the angular distribution to spatial distribution. It is shown that the resolution of this proposed method, to the first order, is independent of the beam's transverse emittance and energy spread. Simulations using LCLS beam confirmed that the method can allow one to measure the temporal profile of an ultrashort electron bunch with a resolution much better than 1 fs.

\section{RESOLUTION LIMIT IN BUNCH LENGTH MEASUREMENT WITH A DEFLECTING CAVITY}

We first go through the principles of bunch length measurement with a deflecting cavity [13]. A deflecting cavity is an $\mathrm{rf}$ structure operating in the TM11 mode. The fast varying field gives the electron beam a time-dependent transverse kick which can be measured in a downstream view screen. The transverse distribution on the screen can be used to calculate the beam's temporal profile.

We assume the bunch goes through the deflecting cavity at a zero-crossing phase and the deflection is in the horizontal plane. We further assume that the bunch length is much shorter than the wavelength of the rf field, which is typical for the $\mathrm{x}$-ray FEL operating parameters. Hereafter we will only consider the dynamics in the $x-z$ plane and neglect the uncoupled motion in the vertical plane. Consider an electron with initial state $\vec{g}_{0}=$ $\left(x_{0}, x_{0}^{\prime}, z_{0}, \delta_{0}\right)^{T}$, where $x_{0}$ is the transverse position, $x_{0}^{\prime}$ is the transverse divergence, and $z_{0}$ and $\delta_{0}$ are relative longitudinal position and energy deviation with respect to the reference particle. After passage through the deflecting cavity, its divergence becomes

$$
x_{1}^{\prime}=x_{0}^{\prime}+k z_{0},
$$

where $k=2 \pi \mathrm{eV} / \lambda E$ is the deflection strength, $V$ is the deflection voltage of the cavity, $E$ is electron energy, and $\lambda$ is the wavelength of the rf field. This time-dependent kick manifests itself in a downstream screen and the transverse distribution can be measured to determine the initial temporal profile. The particle's transverse position on the screen is correlated to its state at the exit of the deflecting 
cavity as $x_{2}=r_{11} x_{1}+r_{12} x_{0}^{\prime}+r_{12} k z_{0}$, where $r_{11}$ and $r_{12}$ are the spatial-to-spatial and angular-to-spatial elements of the transfer matrix from the deflecting cavity to the screen. It is straightforward to show that the transverse size of the beam on the screen is

$$
\sigma_{x}=\sqrt{\sigma_{x 0}^{2}+r_{12}^{2} k^{2} \sigma_{z 0}^{2}}
$$

where $\sigma_{x 0}$ is the beam size on the screen when the deflecting cavity is off.

In order to unambiguously determine the temporal profile of the beam, one may require the beam size on the screen to be dominated by the kick imprinted in the deflecting cavity. Therefore, given the beam parameters and deflecting strength, the minimal bunch length that can be resolved is limited by the intrinsic beam size on the screen. In principle, one can increase the deflecting voltage and reduce the wavelength of the $\mathrm{rf}$ field to increase the resolution. An x-band deflecting cavity is planned at LCLS which is expected to push the resolution to a few femtoseconds [14].

\section{LONGITUDINAL-TO-TRANSVERSE MAPPING}

To overcome the resolution limit, we propose a new scheme with an exact longitudinal-to-transverse mapping as shown in Fig. 1. This mapping system includes a symmetric chicane, an rf deflecting cavity, and a parallel-topoint imaging beam line. The symmetric chicane consists of four dipole magnets (red triangles in Fig. 1) and four quadruple magnets $(\mathrm{Q} 1-\mathrm{Q} 4$, green diamonds in Fig. 1). With properly chosen parameters for the chicane system and the deflecting cavity, the particle's divergence at the exit of the deflecting cavity only depends on its longitudinal position at the entrance to the chicane. The parallel-topoint imaging beam line right after the deflecting cavity including a drift, a quadruple (Q5), and another drift is used to convert the angular distribution to spatial distribution.

We assume the beam is bent in the horizontal plane and only consider the motion in the $x-z$ plane. After passing through a linear Hamiltonian system electron's state $\vec{g}_{1}$ is related to its initial state $\vec{g}_{0}$ by $\vec{g}_{1}=R \vec{g}_{0}$, where $R$ is the symplectic $4 \times 4$ transport matrix of the system. By properly choosing the strength for the quadruples, one can make all the elements $R_{3 i}$ of the chicane transfer matrix vanishing except for $i=2$ and $i=3\left(R_{33}=1\right)$. In this case, due to the symplectic condition and the mirror symmetry, the transfer matrix for the chicane system is [15]

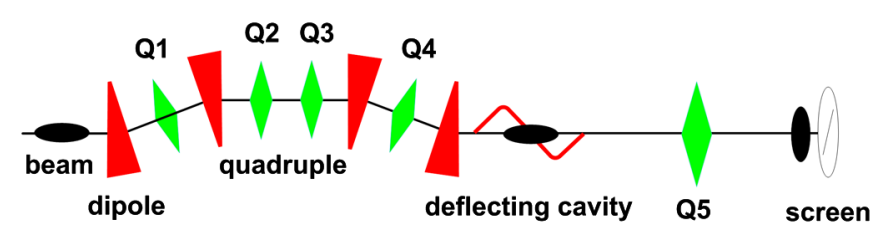

FIG. 1. Scheme for longitudinal-to-transverse mapping.

$$
R_{c}=\left[\begin{array}{cccc}
-1 & R_{12} & 0 & \eta \\
0 & -1 & 0 & 0 \\
0 & -\eta & 1 & 0 \\
0 & 0 & 0 & 1
\end{array}\right]
$$

where $R_{12}$ is the angular-to-spatial element and $\eta$ is the dispersion of the system. The transfer matrix of the deflecting cavity under thin-lens approximation is

$$
R_{d}=\left[\begin{array}{cccc}
1 & 0 & 0 & 0 \\
0 & 1 & k & 0 \\
0 & 0 & 1 & 0 \\
k & 0 & 0 & 1
\end{array}\right]
$$

It is straightforward to obtain the transfer matrix for the chicane and deflecting cavity system,

$$
R_{s}=R_{d} R_{c}=\left[\begin{array}{cccc}
-1 & R_{12} & 0 & \eta \\
0 & -(1+\eta k) & k & 0 \\
0 & -\eta & 1 & 0 \\
-k & k R_{12} & 0 & 1+\eta k
\end{array}\right] .
$$

It turns out that, if $k=-1 / \eta$, the particle's divergence after the deflecting cavity is $x_{1}^{\prime}=k z_{0}$ which only depends on its initial longitudinal position and is independent of the initial transverse position, the initial divergence, and the initial energy deviation.

The parallel-to-point imaging beam line converts the angular distribution to spatial distribution. When the drift length equals the focal length $f$ of the quadruple, the transfer matrix for the parallel-to-point imaging beam line is found to be

$$
R_{b}=\left[\begin{array}{cccc}
0 & f & 0 & 0 \\
-1 / f & 0 & 0 & 0 \\
0 & 0 & 1 & 0 \\
0 & 0 & 0 & 1
\end{array}\right]
$$

Finally, we get the transfer matrix $R$ for the whole longitudinal-to-transverse mapping beam line,

$$
R=R_{b} R_{s}=\left[\begin{array}{cccc}
0 & 0 & f k & 0 \\
1 / f & -R_{12} / f & 0 & 1 / f k \\
0 & 1 / k & 1 & 0 \\
-k & k R_{12} & 0 & 0
\end{array}\right]
$$

It follows from Eq. (7) that, under the condition $k=$ $-1 / \eta$ in this mapping beam line, the final transverse position of the particle is simply $x=f k z_{0}$, which implies that the beam's initial temporal profile is exactly mapped to the beam's final transverse distribution and the effective magnification ratio is $M=f k$. Considering the fact that many devices can measure the transverse beam size with a resolution well beyond $1 \mu \mathrm{m}$ and the magnification ratio can be much larger than unity, one can expect that the proposed technique may have a very high resolution in measuring temporal profiles for ultrashort electron bunches. 


\section{SIMULATIONS}

In the section above for simplicity we only considered the beam dynamics in the $x-z$ plane using the first order transfer matrix. In this section we include the beam dynamics in $6 \mathrm{D}$ phase space. The second order transportation effects and other unwanted effects which may affect the performances of the proposed mapping technique are also included in the simulations. We will discuss the resolution of the proposed technique and present the simulation results using LCLS beam as an example.

\section{A. Resolution of the mapping technique}

The resolution of the proposed technique, to the first order, is independent of transverse emittance and beam energy spread. However, the higher order transportation terms may lead to incomplete mapping, so that the transverse beam size on the screen is nonzero even when the initial bunch length is zero. Similar to the case of a streak camera where the temporal resolution is defined as the impulse response function of the system, the resolution of our mapping system is defined as $\Delta t=\sigma_{x} / M c$, where $\sigma_{x}$ is the rms horizontal size of the beam on the screen when the initial bunch length is zero and $c$ is the speed of light.

For an ultrashort bunch such as that generated at LCLS, the beam typically has small transverse emittance and relatively large energy spread [7]. In this case the resolution is limited by high order chromatic terms rather than high order geometric terms. Specifically, the $T_{266}$ term tends to give the particle an energy-dependent divergence and the $T_{566}$ term gives the particle an energy-dependent longitudinal shift. Such high order correlations cannot be canceled in this system and limit the resolution.

When these two terms dominate, the resolution of this method is approximately

$$
\Delta t \approx \sqrt{2 T_{266}^{2} / k^{2}+2 T_{566}^{2}} \delta_{0}^{2} / c .
$$

Let us consider a beam similar to that in LCLS after the second bunch compressor (BC2). The beam energy is assumed to be $E=4.3 \mathrm{GeV}$ and the normalized projected emittance is assumed to be $0.6 \mathrm{~mm}$ mrad. As for the chicane system, the distances between the quadruples and dipoles are all $4 \mathrm{~m}$. The parameters of the chicane system and the deflecting cavity used in our simulation are listed in Table I. The strength of the quadruples is adjusted to provide vanishing $R_{51}$ and $R_{56}$ (this is achieved with the "optimization" function in ELEGANT code [16]). In this setup the dispersion of the chicane system is $0.62 \mathrm{~m}$. The dominating second order terms are $T_{266}=0.058$ and $T_{566}=0.021 \mathrm{~m}$. The focal length of the parallel-to-point imaging beam line is about $12.4 \mathrm{~m}$ in our simulation so that the magnification ratio is 20 . A crude estimation using Eq. (8) indicates that one can have $0.2 \mathrm{fs}$ resolution if the relative energy spread of the beam is $10^{-3}$.
TABLE I. Main parameters used for the simulations.

\begin{tabular}{lc}
\hline \hline Arc length of the dipole & $0.5 \mathrm{~m}$ \\
Bending angle of the dipole & $20 \mathrm{mrad}^{-2}$ \\
Strength of Q1 and Q4 & $0.33033 \mathrm{~m}^{-2}$ \\
Strength of Q2 and Q3 & $-0.91651 \mathrm{~m}^{-2}$ \\
Length of the quadruples & $0.3 \mathrm{~m}$ \\
Frequency of the deflecting cavity & $11.424 \mathrm{GHz}$ \\
Length of the deflecting cavity & $1.5 \mathrm{~m}$ \\
Voltage of the deflecting cavity & $29 \mathrm{MV}$ \\
\hline \hline
\end{tabular}

We tracked a beam with vanishing initial length through the system and the horizontal beam sizes on the screen are used to determine the resolution in the bunch length measurement. The simulation is performed using ELEGANT code with second order transportation effects included. The initial transverse Twiss parameters are optimized to reduce the second order geometric contribution. Extensive simulations indicate that the second order geometric contribution is more sensitive to the initial Twiss parameters in the vertical plane because the beam is defocused in $y$ by the quadruple for parallel-to-point imaging. If the initial Twiss parameters greatly mismatch with the mapping system, the beta function in the $y$ plane can be very large which will introduce considerable second order geometric aberration. Typically the geometric contribution is much smaller than the chromatic terms. The resolution of this technique for various beam energy spread $\sigma_{\delta}$ is shown in Fig. 2.

From Fig. 2, one can clearly see the quadratic dependence of the resolution on beam energy spread, which implies that the resolution is mainly dominated by second order chromatic terms. In the extreme case when beam energy spread is very small, the resolution is limited by the second order geometric terms, as can be seen in Fig. 2, where Eq. (8) underestimates the initial bunch length when

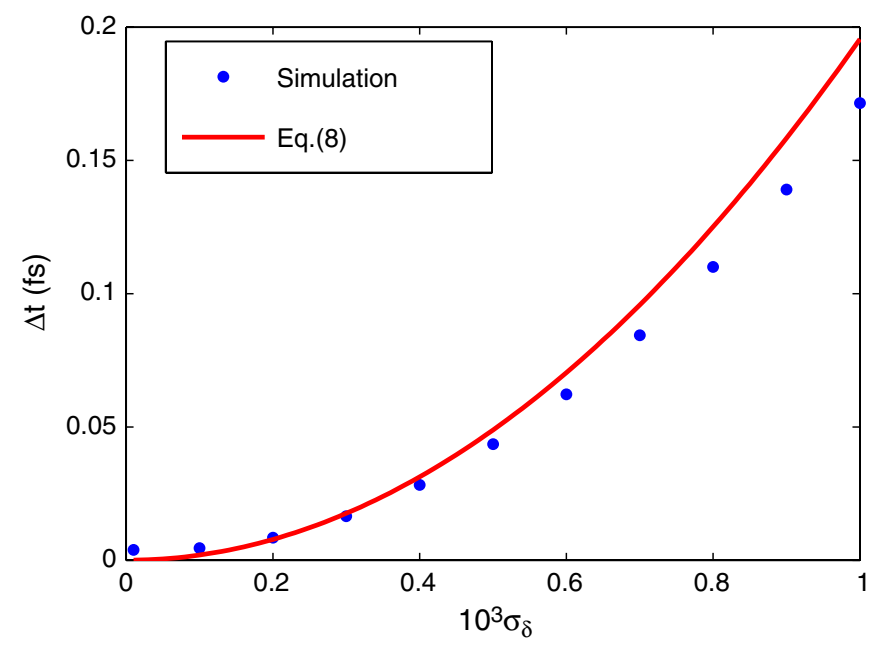

FIG. 2. Resolution in bunch length measurement: blue dots are the resolution determined from the beam size on the screen using ELEGANT simulation and the red curve is the results from Eq. (8). 
beam energy spread is below $2 \times 10^{-4}$ because Eq. (8) neglects the second order geometric terms. It should be pointed out that sextupoles may be used in the chicane to cancel the second order terms and thus improve the temporal resolution. However, a detailed study for this is beyond the scope of this paper.

\section{B. Simulation using LCLS beam}

In the last section we did not include the incoherent synchrotron radiation (ISR) and coherent synchrotron radiation (CSR) effects, so Eq. (8) should be considered as the best resolution one can achieve. In practice, the resolution of the proposed technique can also be affected by ISR and CSR in the chicane. If the particle's energy is changed in a dispersive region, its divergence at the exit of the chicane will be affected due to the nonzero $R_{26}$ term in the transfer matrix and it cannot be canceled with the deflecting cavity. Typically, the CSR can be a main concern for ultrashort bunches. To verify these effects, we performed simulations with the start-to-end simulated LCLS beam dumped after BC2. The bunch charge is $20 \mathrm{pC}$ which is typical for LCLS low charge operation mode. The projected horizontal emittance is about $0.52 \mathrm{~mm}$ mrad and the vertical emittance is about $0.2 \mathrm{~mm}$ mrad. Without using the mapping technique, the temporal resolution is limited to about 3 fs assuming the same parameters for the beam and the deflecting cavity. We will show below that the resolution is significantly improved with the mapping technique.

For a typical LCLS overcompression case, the result is shown in Fig. 3. The predicted temporal profiles are obtained from the horizontal profiles on the screen with a magnification ratio of $M=20$. The predicted temporal profile without considering ISR and CSR (blue dash-dotted line) is in excellent agreement with the original temporal profile (red solid line). When ISR and CSR are taken into account, the temporal profile (magenta dashed line) is shifted because of the net energy loss from CSR and ISR and distorted due to the time-dependent CSR wake. The predicted temporal shape is still in good agreement with the true distribution.

This overcompressed beam has a long tail, so the bunch length is best described by a full width at half maximum (FWHM). The predicted FWHM of the beam including CSR and ISR is $3.07 \mathrm{fs}$ while the true FWHM is $3.52 \mathrm{fs}$. In a separate simulation, we only included the CSR effect and turned off the ISR option in the tracking, and there is no noticeable difference compared with the case when ISR is turned on. However, it should be pointed out that the ISR has a strong dependence on beam energy and bending radius. So it can affect the resolution when beam energy is high and bending radius is small.
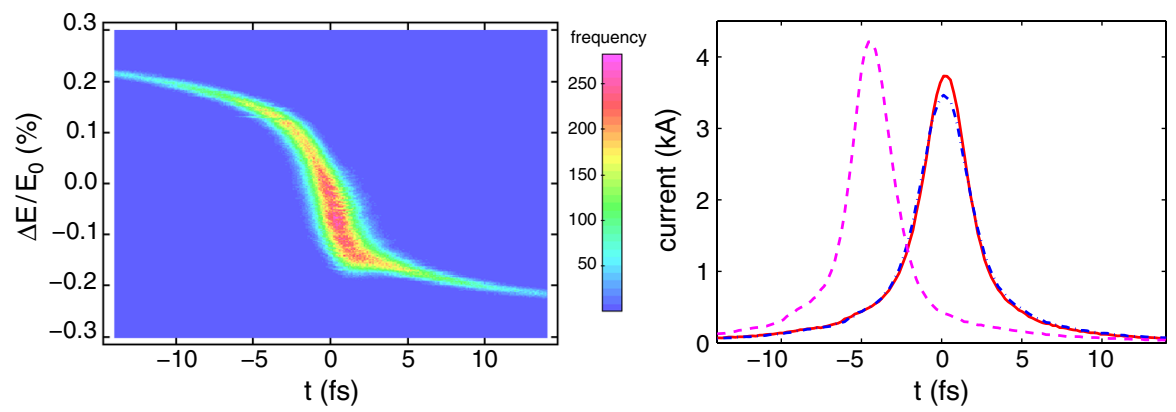

FIG. 3. (left) Longitudinal phase space for the overcompressed beam (bunch head to the left). (right) Temporal profiles: true temporal profile (red solid line); predicted temporal profile without considering ISR and CSR effect (blue dash-dotted line); predicted temporal profile with ISR and CSR taken into account (magenta dashed line).
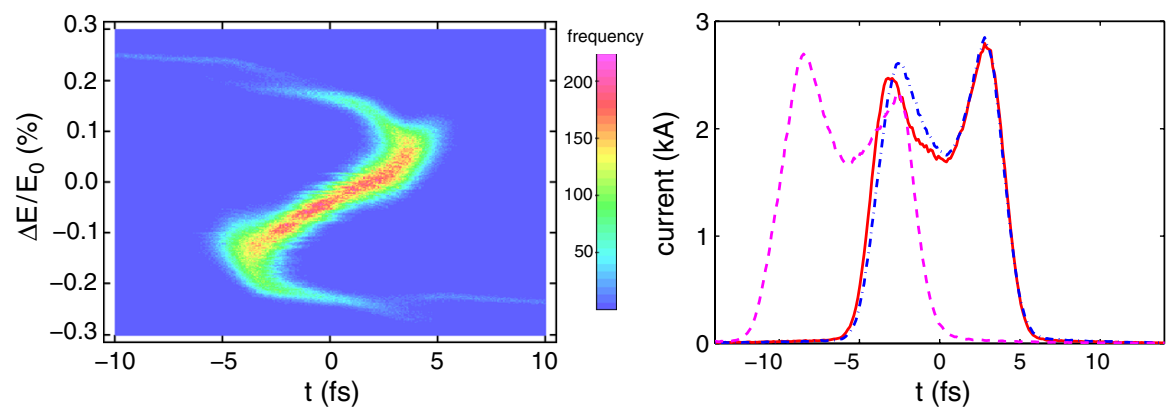

FIG. 4. (left) Longitudinal phase space for the undercompressed beam (bunch head to the left). (right) Temporal profiles: true temporal profile (red solid line); predicted temporal profile without considering ISR and CSR effect (blue dash-dotted line); predicted temporal profile with ISR and CSR taken into account (magenta dashed line). 
A more challenging case is the undercompression mode for LCLS where the beam has a complicated longitudinal phase space distribution and the CSR associated with the double horn structures can be strong. As shown in Fig. 4, a comparison between the blue-dotted curve (without CSR and ISR) and the magenta dash-dotted curve (with CSR and ISR) implies that the distortion is more serious for the tail part of the bunch than the head part. Analysis shows that the bunch shape is modified in the chicane due to longitudinal-and-transverse coupling and the CSR wake for the whole bunch is more pronounced in the tail part, which results in a more serious distortion for the tail part of the bunch. The predicted FWHM bunch length with CSR and ISR taken into account is $8.55 \mathrm{fs}$ while the true rms bunch length is $8.50 \mathrm{fs}$.

In the examples above, the global energy spread of the beam is about $1.1 \times 10^{-3}$ and the estimated resolution is about 0.2 fs. If the chirp associated with the core of the beam is removed, the global energy spread can be reduced and the resolution can be improved. For an ideal LCLS case when the chirp is effectively compensated, the beam has an energy spread of about $1.5 \mathrm{MeV}$, hence the corresponding resolution reaches 20 attoseconds. Alternatively, one can also increase the distances between dipoles and reduce the bending angle and quadruple strength to mitigate the second order effects, which should also improve the resolution.

It is worth mentioning that the condition $k=-1 / \eta$ to achieve longitudinal-to-transverse mapping is the same as that in the emittance exchange (EE) scheme [17-19]. Actually, one can add a dogleg downstream of the deflecting cavity as shown in Fig. 1 to exchange the longitudinaland-transverse emittance. In this case the performances of EE can be significantly improved and this EE scheme is not sensitive to beam energy jitter, because the chicane system is isochronous. It should also be pointed out that the performances of the proposed scheme is not affected by the finite length of the deflecting cavity. When the thicklens matrix for the deflecting cavity is used, we get the same mapping formula for the temporal profile.

\section{CONCLUSIONS}

In conclusion, we proposed a novel scheme to map beam's longitudinal profile exactly to its transverse profile. The method can be used to measure the length and temporal profile for ultrashort electron bunch and the resolution, to the first order, is independent of beam's transverse emittance and energy spread. Simulations using LCLS beam confirmed that the method allows one to measure the temporal profile of an ultrashort electron bunch with a resolution well beyond $1 \mathrm{fs}$. This technique can strengthen our understanding of the physics associated with ultrashort bunch generation and help to realize the future compact FELs that work in low charge mode to deliver ultrashort $\mathrm{x}$ ray pulses.

\section{ACKNOWLEDGMENTS}

We thank M. Borland, A. Chao, P. Emma, Z. Huang, G. Stupakov, M. Woodley, and J. Wu for useful discussions. This work was supported by the U.S. DOE under Contract No. DE-AC02-76SF00515.

[1] X. J. Wang and X. Y. Chang, Nucl. Instrum. Methods Phys. Res., Sect. A 507, 310 (2003).

[2] J. Rosenzweig et al., Nucl. Instrum. Methods Phys. Res., Sect. A 593, 39 (2008).

[3] S. Reiche, P. Musumeci, C. Pellegrini, and J. B. Rosenzweig, Nucl. Instrum. Methods Phys. Res., Sect. A 593, 45 (2008).

[4] I. Blumenfeld et al., Nature (London) 445, 741 (2007).

[5] J. Rosenzweig et al., arXiv:1002.1976v1.

[6] P. Emma, in Proceedings of the 23rd Particle Accelerator Conference, Vancouver, Canada, 2009 (IEEE, Piscataway, NJ, 2009), TH3PBI01.

[7] Y. Ding et al., Phys. Rev. Lett. 102, 254801 (2009).

[8] R. Brinkmann, E. A. Schneidmiller, and M. V. Yurkov, arXiv:1001.3801v1.

[9] S. Reiche, H. Braun, Y. Kim, and M. Pedrozzi, in Proceedings of FEL09 Conference, Liverpool, UK, 2009, p. 59.

[10] H. Loos, in Proceedings of the 23rd Particle Accelerator Conference, Vancouver, Canada, 2009 (Ref. [6]), TU3GRI01.

[11] G. Berden et al., Phys. Rev. Lett. 99, 164801 (2007).

[12] K. Bane et al., Phys. Rev. ST Accel. Beams 12, 030704 (2009).

[13] P. Emma, J. Frisch, and P. Krejcik, Report No. LCLS-TN00-12, 2000 [http://ssrl.slac.stanford.edu/lcls].

[14] J. W. Wang and S. Tantawi, in Proceedings of LINAC 08, Victoria, Canada, 2008, p. 966.

[15] D. Xiang and W. Wan, Phys. Rev. Lett. 104, 084803 (2010).

[16] M. Borland, Advanced Photon Source LS-287, 2000.

[17] M. Cornacchia and P. Emma, Phys. Rev. ST Accel. Beams 5, 084001 (2002).

[18] P. Emma, Z. Huang, K.-J. Kim, and P. Piot, Phys. Rev. ST Accel. Beams 9, 100702 (2006).

[19] D. Xiang, Phys. Rev. ST Accel. Beams 13, 010701 (2010). 